\title{
41. DETERMINATION OF THE MASS OF JUPITER FROM OBSERVATIONS OF 10 HYGIEA DURING 1932-1969
}

\author{
N. S. CHERNYKH \\ Crimean Astrophysical Observatory, Crimea, U.S.S.R.
}

\begin{abstract}
An attempt has been made to derive the mass of Jupiter from modern observations of the minor planet 10 Hygiea. The calculations have been made using computer programmes prepared at the Institute for Theoretical Astronomy. From 250 observations 1932 to 1967 we obtained the reciprocal mass $1047.345 \pm 0.040$ (rms error). After excluding some less reliable observations and allowing for the elliptic aberration terms we obtained 1047.326 \pm 0.033 . By means of a more sophisticated computer programme and utilizing 100 additional observations, some of them made in 1969 , we obtained the value $1047.324 \pm 0.023$. Our results are compared with recent determinations by other investigators.
\end{abstract}

\section{Introduction}

At present the value adopted for the mass of Jupiter is still that obtained by Newcomb and Hill at the end of the last century. Modern authorities are of the opinion that this value for the reciprocal mass, 1047.355, should be increased by about 0.05 (de Sitter, 1938; Clemence, 1949; Kulikov and Subbotina, 1963). Because of the recent overhaul of the whole system of fundamental astronomical constants redetermination of the mass of Jupiter has become a particularly urgent problem. Several astronomers in the United States are following Hill's (1873) plan of improving Jupiter's mass by means of prolonged series of observations of 13 asteroids with mean motions approximately in 2:1 resonance with Jupiter and thus experiencing large long-period perturbations. For some of these asteroids the investigations have already been completed (O'Handley, 1968; Zielenbach, 1969; Klepczynski, 1969; Fiala, 1969).

As was shown by Newcomb (1895), the mass of Jupiter can be reliably determined using less extensive series of observations of some asteroids. Newcomb's well-known value was derived from a discussion of observations of 33 Polyhymnia over a period of $34 \mathrm{yr}$.

In this investigation an attempt is made to derive a value for the mass of Jupiter using modern observations of a minor planet over approximately the same time-span. Modern observations are preferred since they are substantially more accurate than the older, almost exclusively visual, observations, and we have therefore considered only those made during the last four decades.

We wanted to select an asteroid similar to 33 Polyhymnia, i.e., one that is quite bright, has a fairly large eccentricity and small inclination and approaches to within 1.5 AU of Jupiter. We have examined the circumstances of the approaches of several dozen of the brighter minor planets to Jupiter and have checked on the observations available. As a result we found that 10 Hygiea has obvious advantages and therefore selected this planet. It is the brightest object in Hill's list (never fainter than magnitude 
11 at opposition) and has thus been widely observed. During the last four decades 10 Hygiea has made four approaches to Jupiter:

$\begin{array}{ll}1932 \text { December } & 2.16 \mathrm{AU} \\ 1942 \text { May } & 1.73 \mathrm{AU} \\ 1952 \text { September } & 1.55 \mathrm{AU} \\ 1963 \text { February } & 1.79 \mathrm{AU} .\end{array}$

These approaches, recurring with a period of about $10.5 \mathrm{yr}$, bring about considerable perturbations in the planet's orbit: during an interval of $35 \mathrm{yr}$ the daily mean motion changes by $6^{\prime \prime}$, the mean longitude by $2^{\circ}$ and the mean anomaly by $12^{\circ}$.

\section{Observations}

We have collected 410 observations obtained at 29 observatories at 32 oppositions from 1932 to 1969 . This number includes 71 observations made by the author at the Crimean Astrophysical Observatory at five oppositions from 1963 to 1969. Before 1950 there was still a substantial proportion of visual observations. Most of them were recalculated using new positions for the reference stars. The positions of the planet had been determined by the observers relative to star positions in the AGK, the Yale zones, or the Carte du Ciel. Using the tables by Orel'skaya (1962), we reduced all the observations to the common system of the $\mathrm{FK}_{3}$, although as a rule these corrections were not large and thus not of great importance. The times of the observations were reduced to Ephemeris Time and all positions were precessed to the standard equinox 1950.0. About 60 erroneous observations were rejected.

The discussion of the observations was carried out twice and will be described in detail below. In the first solution about 250 observations were treated, 56 of them being visual and the rest photographic. The majority of the observations had accuracies in the range 0 ".5 to $1^{\prime \prime}$. Later we added about 100 observations of fairly high quality.

\section{Numerical Integration. Normal Places for 1932-1967}

As the basis for our calculations we used the following set of elements, obtained by Khanina (1965) from observations 1932-1964:

$$
\begin{aligned}
& \text { Epoch }=1948 \text { July 28.0 ET }=\text { JED } 2432760.5 \\
& M_{0}=257: 032358 \\
& \omega=310.27339 \text { ) } \\
& \Omega=285.37736\} \quad 1950.0 \\
& i=3.81364 \\
& \varphi=5.71756 \\
& n=0.17622832 \\
& a=3.1507861 \mathrm{AU} \text {. }
\end{aligned}
$$


We then integrated the equations of motion of 10 Hygiea from 1931 to 1968 , taking into account the perturbations by Venus to Pluto. This was done with the help of the BESM-2 computer programme by Belyaev (1967), which makes use of a file of osculating elements of these eight planets for 1660-2060. The integration is done by Cowell's method in rectangular coordinates, with 9-10 significant figures and a variable step.

The numerical integration was compared with all the observations 1932 to 1967. Separate observations were then linked into normal places. We formed two normal places for the oppositions of 1957 and 1963 and one for each of the remaining oppositions. Altogether, we formed 28 normal places from 250 observations.

\section{Conditional Equations. Solution Using the Observations 1932-1967}

In addition to the Jupiter mass correction and the usual six unknowns of the problem of improving the orbit of a minor planet, it is possible to include as unknowns corrections to the elements of the Earth's orbit and to the equinox and equator of the system of star positions. In this general case, the conditional equations have the form:

$$
\begin{aligned}
& \cos \delta \sum_{i=1}^{6} \frac{\partial \alpha}{\partial E_{i}} \Delta E_{i}+\cos \delta \sum_{j=1}^{5} \frac{\partial \alpha}{\partial E_{j}^{\prime}} \Delta E_{j}^{\prime}+\cos \delta \frac{\partial \alpha}{\partial m_{5}} \Delta m_{5}=\cos \delta \Delta \alpha_{0} \\
&=\cos \delta(\mathrm{O}-\mathrm{C})_{\alpha} \\
& \sum_{i=1}^{6} \frac{\partial \delta}{\partial E_{i}} \Delta E_{i}+\sum_{j=1}^{5} \frac{\partial \delta}{\partial E_{j}} \Delta E_{j}^{\prime}+\frac{\partial \delta}{\partial m_{5}} \Delta m_{5}+\Delta \delta_{0}=(\mathrm{O}-\mathrm{C})_{\delta},
\end{aligned}
$$

where $E_{i}(i=1,2, \ldots, 6)$ are the elements of the asteroid's orbit, $E_{j}^{\prime}(j=1,2, \ldots, 5)$ are the elements of the Earth's orbit, $m_{5}$ is the mass of Jupiter, and $\Delta \alpha_{0}$ and $\Delta \delta_{0}$ are the equinox and equator corrections. $(\mathrm{O}-\mathrm{C})_{\alpha}$ and $(\mathrm{O}-\mathrm{C})_{\delta}$ are the residuals of the observed positions (normal places) from the positions calculated from the initial conditions. A pair of such equations is formed for each observation or normal place, and the complete solution is made by the method of least squares.

The equations for the 28 normal places of 10 Hygiea contained all 14 unknowns mentioned. The coefficients $\partial \alpha / \partial E_{i}$ and $\partial \delta / \partial E_{i}$ were obtained from the numerical integration, while the same coefficients for the Earth were derived using the rectangular coordinates of the Sun. In order to obtain the coefficients of $\Delta m_{5}$ we made an additional integration using a slightly different value for the mass of Jupiter. All the equations of condition were assigned unit weight.

First of all we just solved for seven of the unknowns: the corrections to the orbital elements of the minor planet and to the mass of Jupiter. The resulting mass of Jupiter was found to be

$$
1 / m_{5}=1047.345 \pm 0.040 \text { (rms error). }
$$

The residuals of some of the normal places were as high as 2 ", with an rms error of unit weight $\sigma_{0}= \pm 0$ ".88. To improve the accuracy we revised the normal places, elimin- 
ating some of the less reliable observations and introducing the elliptic aberration terms. We also solved for all 14 unknowns, but owing to the smallness of the determinant of the coefficients the result was unsatisfactory. This was to be expected because in order to separate the corrections to the elements of the Earth and the asteroid one requires observations made far from opposition, and these were not available. Removal of the correction to the Earth's longitude improved matters, but the corrections to the equinox and to the Earth's remaining elements were still very poorly determined. We could determine with confidence only eight unknowns: the corrections to the elements of the asteroid, to the mass of Jupiter, and to the system of declination. This solution gave for Jupiter's mass

$$
1 / m_{5}=1047.326 \pm 0.033
$$

\section{New Treatment of the Observations}

At this point in our calculations the Institute for Theoretical Astronomy acquired a BESM-4 computer, and two new programmes for numerical integration were written. We decided to perform the calculations anew, using these more accurate programmes and also including a number of additional observations made earlier at the U.S. Naval Observatory and in 1969 at the Crimean Observatory.

Belyaev's new programme has most of the features of the old one, but it can also be used for calculating ephemerides and comparing calculations with observations, and the results are good to one more decimal place. The programme by KazimirchakPolonskaya (1967) has also been revised for the new computer; it makes use of a file of rectangular coordinates of the planets Venus to Pluto for $400 \mathrm{yr}$, and the integration is carried out in double precision using the Numerov-Subbotin method.

Comparison of integrations of the orbit of 10 Hygiea, made using the two new programmes, gave differences in $\alpha$ and $\delta$ of only 0.01 and 0.1 , respectively, after $19 \mathrm{yr}$. The new calculation had an insignificant effect on the residuals.

Inclusion of a large number of additional observations from 1956 to 1967 allowed us to form more normal places. The total number of observations was increased to 350 , and we formed 37 normal places. With few exceptions, the distribution of observations in the normal places became more uniform.

The coefficients in the conditional equations were calculated as before, and the equations were all given unit weight, but in view of the unsatisfactory results obtained when we solved for corrections to the Earth's orbit, we decided to retain seven unknowns only. The new solution proved to be very similar to the previous one. In particular, the reciprocal mass of Jupiter was

$$
1 / m_{5}=1047.324 \pm 0.023 \text {. }
$$

The difference between the two values is negligible, and even our preliminary result of 1047.345 is contained within the error limits.

Table I shows several recent determinations of the mass of Jupiter using minor planets and satellites, the results being listed in order of increasing mass. These 
TABLE I

Modern determinations of the mass of Jupiter

\begin{tabular}{llll}
\hline $\begin{array}{l}1 / m_{5} \text { and mean } \\
\text { error }\end{array}$ & Reference & Object & Note \\
\hline $1047.387 \pm 0.004$ & O'Handley (1968) & $\begin{array}{l}\text { 65 Cybele } \\
\text { Jupiter IX }\end{array}$ & \\
$1047.386 \pm 0.041$ & Bec (1969) & 52 Europa & $(1)$ \\
$1047.381 \pm 0.020$ & Klepczynski (1967) & 31 Euphrosyne & \\
$1047.372 \pm 0.006$ & Klepczynski (1969) & 57 Mnemosyne & $(2)$ \\
$1047.367 \pm 0.004$ & Fiala (1969) & 24 Themis & \\
$1047.359 \pm 0.010$ & Klepczynski (1969) & 57 Mnemosyne & $(2)$ \\
$1047.356 \pm 0.004$ & Fiala (1969) & 10 Hygiea & \\
$1047.351 \pm 0.006$ & Klepczynski (1969) & 57 Mnemosyne & $(2)$ \\
$1047.350 \pm 0.004$ & Klepczynski (1969) & 10 Hygiea & $(3)$ \\
$1047.345 \pm 0.040$ & This investigation & 48 Doris & $(4)$ \\
$1047.340 \pm 0.024$ & Zielenbach (1969) & 52 Europa & $(1)$ \\
$1047.337 \pm 0.027$ & Klepczynski (1969) & Jupiter VIII & \\
$1047.335 \pm 0.077$ & Herget (1968) & 48 Doris & $(4)$ \\
$1047.333 \pm 0.024$ & Zielenbach (1969) & 10 Hygiea & $(3)$ \\
$1047.326 \pm 0.033$ & This investigation & 10 Hygiea & $(3)$ \\
$1047.324 \pm 0.023$ & This investigation & & \\
\hline
\end{tabular}

(1) From 297 and 561 observations, respectively.

(2) The first value is from 986 observations reduced to the $\mathrm{FK}_{4}$ and the second included additional observations; the third value is also by Fiala, quoted by Klepczynski.

(3) The first value is preliminary.

(4) For the second value the differential coefficients for the orbital corrections were obtained from variational equations.

determinations are all grouped near the Newcomb-Hill value, suggesting that the correction indicated by de Sitter and by Clemence is unwarranted. We may note also that Klepczynski's result using 10 Hygiea is on the same side of the Newcomb-Hill figure as ours.

\section{Acknowledgments}

I wish to express my cordial thanks to N. S. Yakhontova for her constant attention and to N. A. Belyaev and E. I. Kazimirchak-Polonskaya for their help with the calculations.

\section{References}

Bec, A.: 1969, Astron. Astrophys. 2, 381.

Belyaev, N. A.: 1967, Byull. Inst. Teor. Astron. 10, 696.

Clemence, G. M.: 1949, Proc. Am. Phil. Soc. 93, 7.

de Sitter, W.: 1938, Bull. Astron. Inst. Neth. 8, 307.

Fiala, A. D.: 1969, Bull. Am. Astron. Soc. 1, 342.

Herget, P.: 1968, Astron. J. 73, 737.

Hill, G. W.: 1873, Collected Mathematical Works, Vol. I.

Kazimirchak-Polonskaya, E. I.: 1967, Trudy Inst. Teor. Astron. 12.

Khanina, F. B.: 1965, Byull. Inst. Teor. Astron. 10, 424.

Klepczynski, W. J.: 1967, Astron. J. 72, 808. 
Klepczynski, W. J.: 1969, Astron. J. 74, 774.

Kulikov, D. K. and Subbotina, N. S.: 1963, in Problemy Dvizheniya Iskusstvennykh Nebesnykh Tel, Moscow.

Newcomb, S.: 1895, Astron. Pap. Washington 5, part 5.

O'Handley, D. A.: 1968, Astron. J. 73, 529.

Orel'skaya, V. I.: 1962, Byull. Inst. Teor. Astron. 8, 660.

Zielenbach, J. W.: 1969, Astron. J. 74, 567.

\section{Discussion}

W. J. Klepczynski: I wish to congratulate you on this fine work. I agree that Newcomb's mass of Jupiter is about the best so far, and I think that any further improvement will have to come from the close approach of a space probe to Jupiter. 